\title{
Computed tomography abnormalities antedating mesothelioma diagnosis: a perspective on the natural history
}

\author{
To the Editor:
}

Malignant pleural mesothelioma (MPM) is an aggressive and fatal disease that typically presents with breathlessness, chest pain or both [1]. It is usually a unilateral disease but $3 \%$ of patients have malignant disease bilaterally at presentation [2]. The latency period between exposure to asbestos and MPM development is $20-40$ years.

Computed tomography (CT) is the best imaging modality to assess for malignant pleural involvement [2]. Involvement of inter-lobar fissures and circumferential pleural involvement with hemithorax contraction suggest MPM [3, 4], but neither of these signs is specific enough to rule out other causes of pleural malignancy. In addition, tumour thickness measured by CT is the standard method to measure MPM response to therapy, although CT tumour volumetry is another method under investigation [5]. Patients with MPM frequently, but not invariably, have CT evidence of other asbestos-related pleural disease. These include plaques, diffuse thickening and effusion. The frequency of these abnormalities and their distribution in relation to MPM is not well characterised. Additionally, CT has a low negative predictive value in asbestos-exposed patients who present with a bland-looking effusion; often a pleural biopsy is needed to differentiate MPM from benign asbestos-related effusion [6]. We aimed to study chest CT findings in scans conducted before a diagnosis of MPM was made to learn more about the evolution of MPM, and the natural history of early radiological features, with a view to identifying radiological change which was potentially "at risk" of later progression to mesothelioma.

In a retrospective study of the local database of mesothelioma patients diagnosed at Oxford University Hospitals (Oxford, UK) between 2009 and 2017, patients' electronic records were screened for chest CT scans before the diagnostic CT that suggested a diagnosis of mesothelioma. Patients' clinic letters were examined to extract details about asbestos exposure, concurrent malignancy and mesothelioma tissue type. The following radiological details were sought: the indication for each scan and the presence of pleural abnormalities (namely, effusion, thickening and plaques). CT scans were grouped into three time periods: within 1 year prior to the diagnostic CT scan (period A); 1-3 years before diagnosis (period B); and $>3$ years before diagnosis (period $\mathrm{C}$ ).

Out of 190 patients in the database, 47 had CT scans antedating the diagnosis of MPM. 11 (23.4\%) patients were diagnosed on a clinico-radiological basis either due to repeated negative biopsies or frailty precluding any invasive diagnostic testing. 36 (76.6\%) patients were histologically confirmed (26 epithelioid, six biphasic, three sarcomatoid and one desmoplastic pathology). The median (interquartile range) age of patients was 80 (69-84) years, and 11 patients (23.4\%) had history of previous unrelated malignancy. 39 (82.9\%) patients were males, and previous asbestos exposure was documented in $32(68 \%)$ patients. The mean $(95 \% \mathrm{CI})$ time from diagnosis to death in this cohort was $17.8(11-24)$ months. For the epithelioid histology, mean survival was 22.8 (14-31) months, for biphasic, it was $11.2(4.8-17.5)$ months and for clinical diagnosis it was 4.4 (1.9-7.6) months.

76 pre-diagnosis CT scans were available for analysis. The mean time between a CT scan and diagnosis was 18 months (range 12-36 months; minimum 5 months, maximum 109 months). 21 scans (for 18 patients)

@ERSpublications

Radiological evolution of MPM is difficult to infer. Previous pleural effusion or thickening warrants careful follow-up. Some patients don't exhibit abnormalities a few months prior to diagnosis while others carry pleural abnormalities for several years. http://ow.ly/uTGO30mJ8D2

Cite this article as: Hassan M, Tsikrika S, Asciak R, et al. Computed tomography abnormalities antedating mesothelioma diagnosis: a perspective on the natural history. Eur Respir J 2019; 53: 1800935 [https://doi.org/10.1183/13993003.00935-2018]. 

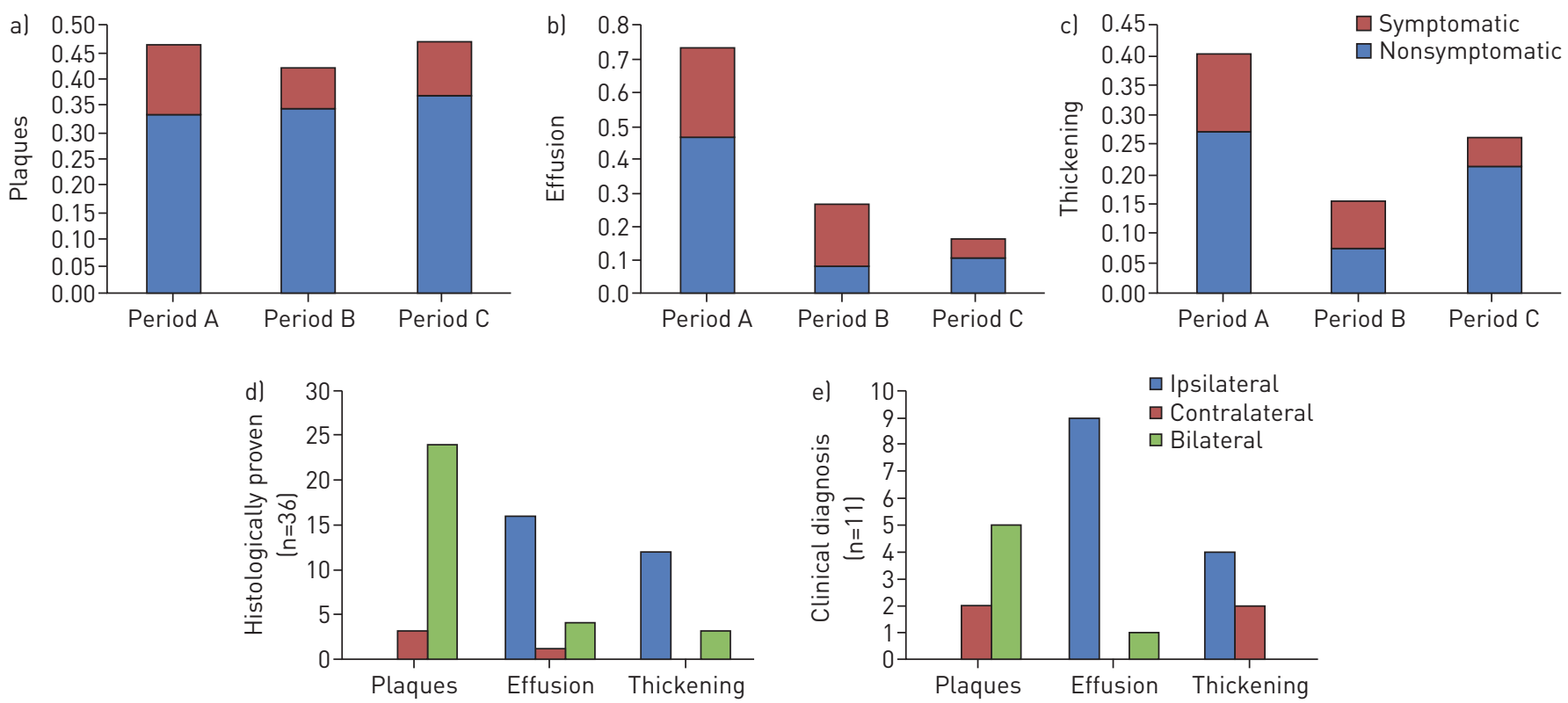

FIGURE $1 \mathrm{a}-\mathrm{c}$ ) Proportion of different pleural abnormalities in pre-diagnosis computed tomography (CT) scans in the three time periods for histologically proven cases. The portion of the CT scans performed on symptomatic patients is presented in red. d and e) The relationship between the side of pre-diagnosis abnormality and the side of mesothelioma development in histologically proven and clinically diagnosed cases.

from period A, 32 scans (for 25 patients) from period B and 23 scans (for 21 patients) from period C were analysed.

Abnormal pleural findings were seen in $58(76 \%)$ scans (in 45 out of 61 scans of histologically proven cases and in 13 out of 15 scans of clinically diagnosed cases). These were divided between the three periods as follows: 20 out of $21(95 \%)$ were positive in period A; 23 out of $32(72 \%)$ were positive in period B; and 15 out of $23(65 \%)$ were positive in period C. Figure 1a demonstrates the prevalence of different abnormalities in the three time periods for the histologically proven cases. A similar pattern was seen in the scans from patients with a clinical diagnosis. Figure $1 \mathrm{~b}$ shows the side of the abnormality in comparison to the side of eventual disease development. Pleural plaques were more commonly seen in patients with previous documented asbestos exposure in similar proportions in both clinical and histologically diagnosed cases (present in $60 \%$ with positive exposure versus $23 \%$ of those with negative history). No considerable differences were noted in effusion and thickening.

Information on the indication for the scans was available in 72 of the 76 scans and the most common were extrapleural cancer staging or follow-up (16 scans, 22.2\%), lung abnormality on chest radiography (15 scans, 20.8\%) and persistent respiratory symptoms (14 scans, 19.4\%). Patients complained of symptoms consistent with pleural disease in $20(28 \%)$ scans. The relation between symptoms and abnormality was strongest in cases of effusion. 15 (65\%) of the 23 patients with pleural effusion were symptomatic, whereas $14(35 \%)$ of the 40 patients without effusion were symptomatic (Chi-squared 5.3, asymptotic significance 0.021 ). The relationship between symptoms and both thickening and plaques was weaker. There was no appreciable relationship between the subtype of MPM and the reported asbestos exposure or the pattern of CT abnormality.

The study cohort shows the typical demographic and clinical characteristic for MPM patients. The high prevalence of clinical diagnosis $(23.4 \%)$ points to the late presentation of the disease in some patients and the challenge in obtaining diagnostic biopsies in others [2].

MPM development is causally related to asbestos exposure, and it is specifically seen in patients exposed at an early age [1]. However, it has been reported in patients without previous documented exposure [7]. The cumulative dose of exposure is not crucial to the development of MPM, as is the case with benign asbestos disease $[1,8]$. In this study, $32 \%$ of patients did not report exposure to asbestos, which could be due to brief exposures that were subject to recall bias. The finding that pleural plaques were more commonly seen in those with documented exposure lends support to the notion that the development of plaques is related to long exposures.

The feasibility and usefulness of screening for early MPM is questionable [7]. In part, this is because the early appearances of mesothelioma on thoracic CT are not well characterised [9]. Additionally, it is not 
known if early discovery of the disease affects prognosis [7]. While a large screening study of $>1000$ patients using low-dose CT failed to discover any MPM [10], another study demonstrated several new diagnoses of MPM on follow-up by observing for changing plaque morphology [9]. It was noted that in workers exposed to asbestos, and even after controlling for length of asbestos exposure, the presence of plaques was associated with an increased statistical risk of developing MPM [11]. However, the agreement in the mesothelioma community is that plaques are mostly regarded as a benign condition unrelated to MPM, and a marker of previous asbestos exposure $[2,12]$. The other benign pleural manifestations (effusion and thickening) are also generally regarded as not associated with increased risk of MPM [8].

By comparing the side with the previous abnormality and the side with MPM development, the data in this study are concordant with the general agreement that plaques are not related to MPM development. A strong signal, however, was demonstrated between historical pleural effusion and MPM development. Pleural thickening was noted to be generally seen on the side of MPM development. Both effusion and thickening were noted on scans that predated diagnosis of MPM by more than a year.

In conclusion, it is difficult to infer a pattern of evolution of MPM from our data, but the presence of previous pleural effusion or thickening warrants careful follow-up. A few patients do not exhibit any abnormalities a few months before diagnosis while others carry pleural abnormalities for several years prior to a diagnosis of mesothelioma. This information might be of clinical relevance as lung cancer screening programmes, and CT scans in general, are used more widely across the UK.

Maged Hassan $\oplus^{1,2,3}$, Stamatoula Tsikrika ${ }^{4}$, Rachelle Asciak $^{1,2}$, Rachel M. Mercer ${ }^{1,2}$, Anwar El-Ganady ${ }^{3}$ and Najib M. Rahman ${ }^{1,2,5}$

${ }^{1}$ Oxford Pleural Unit, Oxford University Hospitals, Oxford, UK. ${ }^{2}$ Oxford Respiratory Trials Unit, University of Oxford, Oxford, UK. ${ }^{3}$ Chest Diseases Dept, Faculty of Medicine, Alexandria University, Alexandria, Egypt. ${ }^{4}$ Pulmonary Division, Dept of Critical Care, Medical School, National and Kapodistrian University of Athens, Evangelismos General Hospital, Athens, Greece. ${ }^{5}$ Oxford NIHR Biomedical Research Centre, Oxford, UK.

Correspondence: Maged Hassan, Oxford Centre for Respiratory Medicine, Churchill Hospital, Old Road, OX3 7LE, Oxford, UK. E-mail: magedhmf@gmail.com

Received: May 182018 | Accepted after revision: Nov 022018

Conflict of interest: None declared.

Support statement: M. Hassan is a recipient of the European Respiratory Society Long-term Research Fellowship - ERS LTRF 2016 -7333. N.M. Rahman is funded by the Oxford NIHR Biomedical Research Centre.

\section{References}

Antman KH. Natural history and epidemiology of malignant mesothelioma. Chest 1993; 103: 373S-376S.

2 Woolhouse I, Bishop L, Darlison L, et al. British Thoracic Society Guideline for the investigation and management of malignant pleural mesothelioma. Thorax 2018; 73: i1-i30.

3 Sahin AA, Cöplü L, Selçuk ZT, et al. Malignant pleural mesothelioma caused by environmental exposure to asbestos or erionite in rural Turkey: CT findings in 84 patients. Am J Roentgenol 1993; 161: 533-537.

4 Roach HD, Davies GJ, Attanoos R, et al. Asbestos: when the dust settles - an imaging review of asbestos-related disease. RadioGraphics 2002; 22: S167-S184.

5 Armato SG, Coolen J, Nowak AK, et al. Imaging in pleural mesothelioma: a review of the 12th International Conference of the International Mesothelioma Interest Group. Lung Cancer 2015; 90: 148-154.

6 Blyth KG, Murphy DJ. Progress and challenges in mesothelioma: from bench to bedside. Respir Med 2018; 134: $31-41$.

7 Scherpereel A, Astoul P, Baas P, et al. Guidelines of the European Respiratory Society and the European Society of Thoracic Surgeons for the management of malignant pleural mesothelioma. Eur Respir J 2010; 35: 479-495.

8 Reid A, de Klerk N, Ambrosini G, et al. The additional risk of malignant mesothelioma in former workers and residents of Wittenoom with benign pleural disease or asbestosis. Occup Environ Med 2005; 62: 665-669.

9 Roberts HC, Patsios DA, Paul NS, et al. Screening for malignant pleural mesothelioma and lung cancer in individuals with a history of asbestos exposure. J Thorac Oncol 2009; 4: 620-628.

10 Fasola G, Belvedere O, Aita M, et al. Low-dose computed tomography screening for lung cancer and pleural mesothelioma in an asbestos-exposed population: baseline results of a prospective, nonrandomized feasibility trial an Alpe-Adria Thoracic Oncology Multidisciplinary Group Study (ATOM 002). Oncologist 2007; 12: 1215-1224.

11 Pairon J-C, Laurent F, Rinaldo M, et al. Pleural plaques and the risk of pleural mesothelioma. JNCI J Natl Cancer Inst 2013; 105: 293-301.

12 Maxim LD, Niebo R, Utell MJ. Are pleural plaques an appropriate endpoint for risk analyses? Inhal Toxicol 2015; 27: 321-334. 